\title{
The Finite Element Analysis of the Vacuum Magnetron Sputtering Coating Chamber
}

\author{
Jie $\mathrm{Wu}^{1, \mathrm{a}}$, shanpeng Sun ${ }^{1, \mathrm{~b}}$ and Dongzheng Wang ${ }^{2, \mathrm{c}}$ \\ ${ }^{1}$ School of Mechanical Engineering, Shenyang University, Liaoning 110044, China \\ ${ }^{2}$ Faculty of Electronic information and Electrical Engineering, Dalian University of Technology, \\ Liaoning 116024, China \\ awujied85@163.com, b1852609778@qq.com, c418731866@qq.com
}

Keywords: Vacuum magnetron sputtering, Coating chamber, Finite element analysis.

\begin{abstract}
In this paper, the vacuum magnetron sputtering coating chamber was as the research object. The three-dimensional model of the coating chamber shell was established by using Sold Works software. The model of the coating chamber shell was imported into the ANSYS finite element software. The finite element model was established and carried on the static analysis. The coating chamber structure improved is proposed, improved coating chamber shell after the design has obviously improved in stability and reliability, to provide theoretical basis for design.
\end{abstract}

\section{Introduction}

The coating chamber housing is a key component of magnetron sputtering coating equipment. Its performance should meet the requirements of the basic process, and to ensure that the substrate holder in interior can stable operation, the requirements of indoor temperature uniformity, good shielding effect, uniform flow, in order to realize continuous plating. So the design of shell structure should follow the minimum deformation, symmetry and simple structure, good sealing performance, convenient opening, easy installation features of target. Magnetron sputtering vacuum magnetron sputtering coating chamber for the design of the main hemisphere samples, the magnetron target on the side and in the formation of sputtering.

Vacuum magnetron sputtering coating machine is composed of vacuum chamber main body system, roasting and heating system, sample system, the magnetron target adjustment system, baffle system, window system, and the glove box system. Vacuum coating chamber is a typical pressure vessel; vacuum magnetron sputtering coating equipment room in addition to the small bell coating chamber structure, the majority of coating equipment adopts a cylindrical or rectangular metal welding coating chamber structure. The structure of the coating chamber should be easy to manufacture and assembly of the internal components, work should be easy to operate, especially in the hot working conditions should have good stability, rigidity, and prevent excessive stress and deformation.

\section{The Vacuum Magnetron Sputtering Coating Chamber Structure}

The vacuum vessel is mainly under atmospheric pressure, the maximum pressure difference is equivalent to a high pressure. Because the vacuum vessel wall is far less than the size of the vacuum vessel, so the vacuum chamber are thin-walled vessels subjected to external pressure, the strength calculation according to the thin shell theory and the conditions to determine under external pressure. The vacuum magnetron sputtering coating chamber is a rectangular metal welding structure. The structure of vacuum magnetron sputtering coating chamber is shown in Figure 1. 


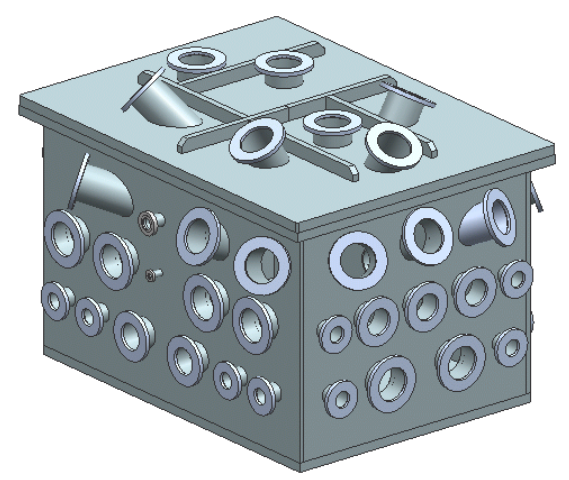

Fig1 The3D model of the coating chamber

\section{The Finite Element Analysis of Vacuum Magnetron Sputtering Coating Chamber}

There are much finite element analysis software now. Such as: NASTRAN, ANSYS, ABAQUS and AFEMS. In this article ANSYS finite element software is used. The three-dimensional model of the coating chamber shell was established by using Sold Works software. The chamber body are stainless 0Cr18Ni9 (SUS304). The chamber body main dimensions and material properties such as table 1.

Table1 Main Dimensions of Vacuum Magnetron Sputtering Coating Chamber

\begin{tabular}{lcc}
\hline \multicolumn{1}{c}{ Projects } & Value & Unit \\
\hline Thickness of coating chamber & 14 & $\mathrm{~mm}$ \\
Total Length of coating chamber & 1218 & $\mathrm{~mm}$ \\
Total Height of coating chamber & 780 & $\mathrm{~mm}$ \\
Total width of coating chamber & 918 & $\mathrm{~mm}$ \\
allowable bending stress of material $([\sigma])$ & 260 & $\mathrm{Mpa}$ \\
Density & 7930 & $\mathrm{Kg} / \mathrm{m}^{3}$ \\
The Elastic Modulus (E) & 193 & $\mathrm{GPa}$ \\
Poisson's Ratio (u) & 0.3 & \\
\hline
\end{tabular}

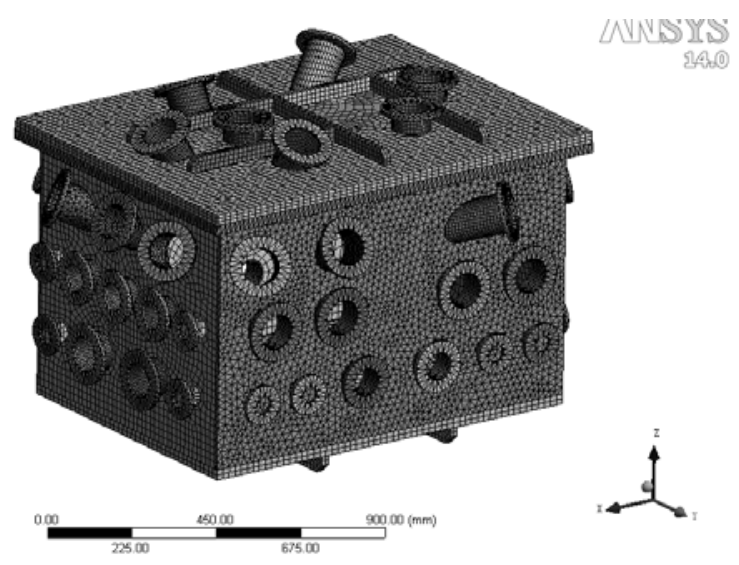

Fig2 FEA model of coating chamber

The vacuum magnetron sputtering coating chamber model meshing is shown as Figure2, a total of 435821 nodes, 118219 units. The applied load method is the finite element model of direct load method, according to the actual work of magnetron sputtering in vacuum chamber, the vacuum chamber in the work load, the main production: gravity and atmospheric pressure on the surface of the vacuum chamber. The total weight of the vacuum chamber of the component is $927.65 \mathrm{~kg}$. The vacuum chamber is mainly under atmospheric pressure, the maximum pressure difference is equivalent to a high pressure, which is subjected to external pressure is 101325Pa. The vacuum 
chamber is thin-walled vessels subjected to external pressure, the strength calculation according to the thin shell theory and the conditions to determine under external pressure.

In the finite element to establish the correct vacuum magnetron sputtering coating chamber model of the structural static analysis, the static structure analysis by ANSYS, can get the whole structure under static load, deformation, strain and stress of the coating chamber data such as shown in table 1.

The contour plot of displacement distribution of the main components of the coating chamber is shown as in Figure3.The contour plot of strain distribution of the main components of the coating chamber is shown as in Figur4. The contour plot of stress distribution of the main components of the coating chamber is shown as in Figur5. The contour plot of displacement distribution of the upper cover ing chamber chamber is shown as in Figur6. The contour plot of strain distribution of the upper cover of the coating chamber is shown as in Figur7. The contour plot of stress distribution of the upper cover of the coating chamber is shown as in Figur8.

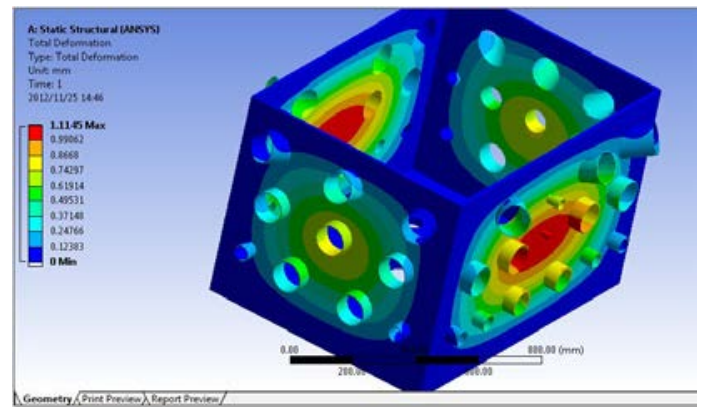

Fig. 3 The Displacement Deistribution of the Main Components

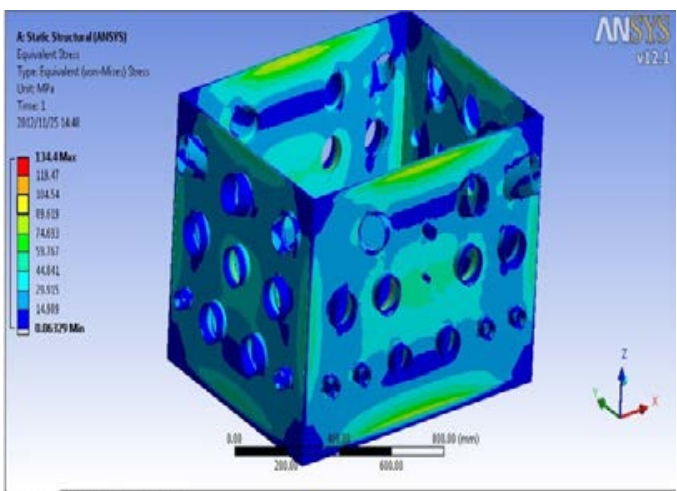

Fig. 5 The Stress Distribution of the Main Components

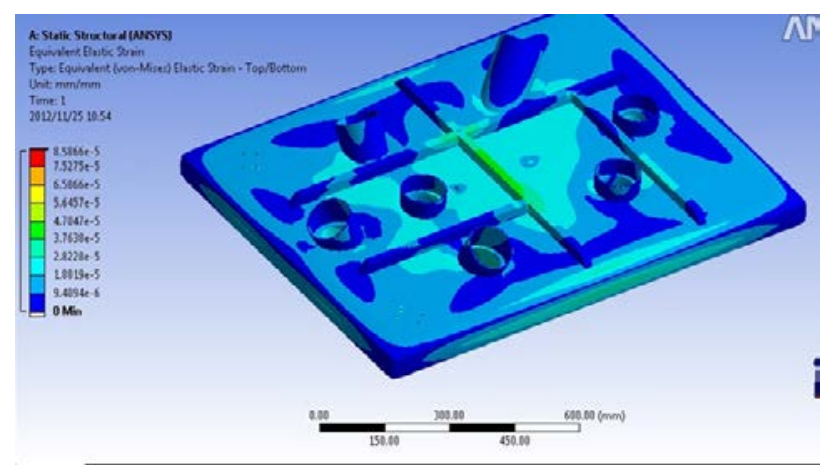

Fig. 7 The Strain Distribution of the Upper Cover ing Chamber Chamber

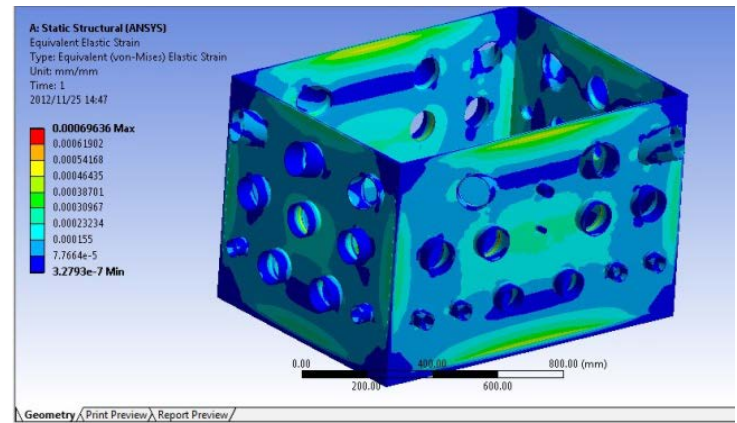

Fig. 4 The Strain Distribution of the MainComponents

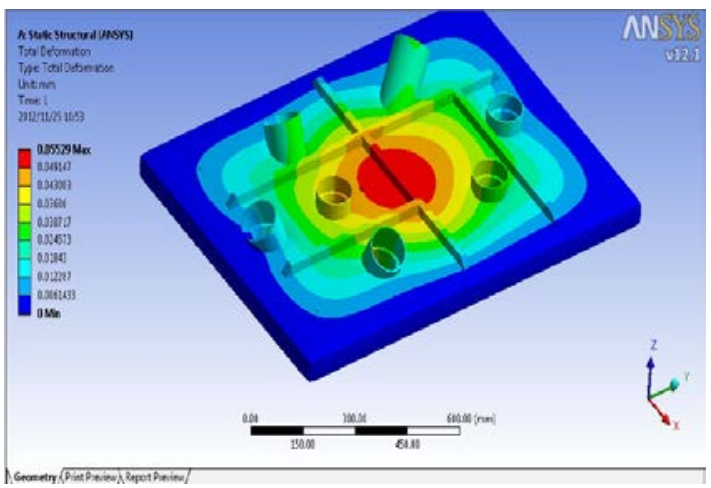

Fig. 6 The Displacement Distribution of the Upper Cover ing Chamber Chamber

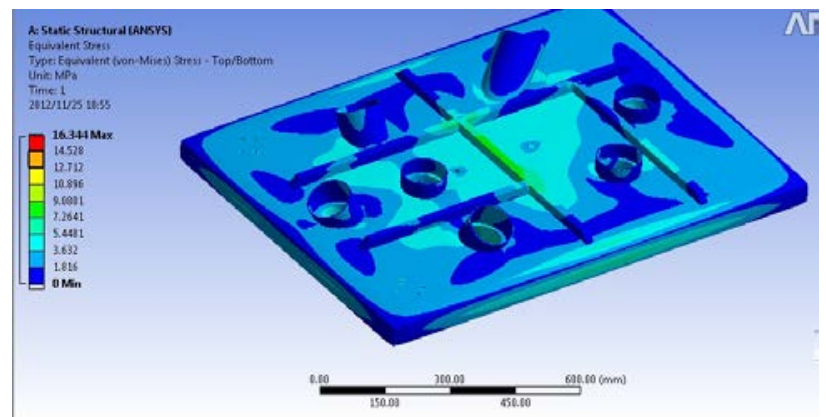

Fig. 8 The Stress Distribution of the Upper Cover ing Chamber Chamber 


\section{Conclusion}

The main components of the coating chamber stress analysis results can be seen, vacuum shell maximum stress in the shell side of the center position of the long side, the maximum stress value is 134Mpa, the coating chamber strength to meet the design requirements, the working process of the coating chamber, static strength can meet the system requirements for the work.

This article introduced the structure design of the Vacuum Magnetron Sputtering Coating Chamber, and Sold Works software for 3D entity modeling, and then conducted on the physical model of reasonable simplification, select the unit type finite element analysis, meshing, set up the finite element model of the Vacuum Magnetron Sputtering Coating Chamber, after applying constraints and loads, the static model, according to the result of model calculation, to analyze the structural is verified. References

\section{References}

[1] ChristoPh M. Constraint-Based Computer-Aided Design .Journal of computing and Information Science in Engineering. Vol. 23 (2005), p. 182-185.

[2] S.J. Lee. Capitation detection of butter fly valve using support vector machines. Journal of sound and Vibration. Vol. 65 (2012), p. 123-126.

[3] Jie Wu, Zhaomeng Yang, and DongZheng Wang et al. The Finite Element Analysis of the Large-Diameter Composite Slurry. Advanced Materials Research. Vols. 989-994 (2014) pp 3149-3152. 